\title{
COVID-19 a substantial burden for hospitals and patients in the USA
}

COVID-19 results in a significant burden in terms of hospital costs and length of stay (LOS) in the USA, according to the results of an analysis published in Advances in Therapy. ${ }^{*}$

The US-based researchers used COVID-19 hospital admissions data from the Premier Healthcare Database to estimate hospital costs, LOS and discharge status for adult COVID-19 patients hospitalised in the US from 1 April 1 to 31 December 2020. Monthly trends in costs, LOS, and discharge status were assessed, with results stratified by patient and hospital characteristics, levels of hospitalisation care, and discharge status; regression analyses were used to estimate factors associated with changes in these parameters.

Of the 247590 hospitalised COVID-19 patients included in the study, 49\% were women, 76\% were aged $\geq 50$ years, and $36 \%$ were admitted to intensive care units (ICU). The median hospital LOS was 6 days, median costs were $\$$ US11 276, and the cost per day was \$1772, respectively. For patients admitted to the ICU, the median LOS was 5 days at a cost of $\$ 13443$ and a cost per day of $\$ 2902$. Patients on mechanical ventilation had the highest hospital and ICU costs (\$47 454 and \$41 510, respectively) and LOS (16 and 11 days). Fourteen percent of patients died in hospital and $52 \%$ were discharged. The main drivers of costs, LOS and risk of death were older age, Black and Caucasian race, hypertension and obesity, and treatment with extracorporeal membrane oxygenation, and discharge to long-term care facilities. The authors note that there were significantly lower median hospital and ICU costs, and LOS in December compared with April.

* The study was funded by Eli Lilly and Company.

Ohsfeldt RL, et al. Inpatient Hospital Costs for COVID-19 Patients in the United States. Advances in Therapy : 5 Oct 2021. Available from: URL: https://doi.org/10.1007/ s12325-021-01887-4 